\title{
Alternativas de enseñanza adoptadas por los profesores universitarios para motivar el aprendizaje en los contextos de aula
}

\author{
Teaching alternatives adopted by \\ university professors to motivate \\ learning in classroom contexts
}

\author{
http://dx.doi.org/10.17981/cultedusoc.13.1.2022.09
}

Recibido: 2 de enero de 2021. Aceptado: 10 de mayo de 2021. Publicado: 24 de enero de 2022.

\author{
Benigno Benavides-Martínez \\ Universidad Autónoma de Nuevo León. Monterrey (México) \\ benignobenavides@gmail.com
}

Para citar este artículo:

Benavides-Martínez, B. (2022). Conocimientos, prácticas y conductas sexuales en educación superior. Cultura, Educación y Sociedad, 13(1), 143-160. DOI: http://dx.doi.org/10.17981/cultedusoc.13.1.2022.09

Resumen

Introducción: Este artículo se enfoca en el estudio de la enseñanza universitaria, específicamente analiza las decisiones tomadas por el profesor en su rol de enseñanza, ante las expectativas formales de los actores educativos y las condiciones concretas del aula, las cuales suelen distanciarse en mayor o menor medida. El profesor se coloca en el centro de la toma de decisiones de las acciones docentes, pero debe seleccionar los cambios pertinentes que incidan positivamente en el aprendizaje. Metodología: Se basa en la construcción de categorías relativas al análisis de las expectativas y las formas seleccionadas por los profesores para despertar y mantener la atención de los estudiantes, así como para reconocer sus diferencias individuales dentro y fuera del aula. La información se obtuvo a través de entrevistas aplicadas en la Universidad Autónoma de Nuevo León (México). Resultados y Discusión: Los profesores son quienes toman las decisiones de enseñanza en el aula, identificando la motivación como uno de los factores de mayor motricidad en el sistema. Conclusiones: Resalta la importancia de resignificar los procesos educativos en correspondencia con los perfiles de los actores.

Palabras clave: Docencia universitaria; toma de decisiones; expectativas de aprendizaje; motivación; significado de la enseñanza

\section{Abstract}

Introduction: This article focuses on the study of university education, specifically analyzes the decisions made by the teacher in their teaching role, in the face of the formal expectations of the educational actors and the concrete conditions of the classroom, which tend to distance themselves. To a lesser extent the teacher is placed at the center of the decisionmaking of teaching actions but must select the relevant changes that positively affect learning. Methodology: It is based on the construction of categories related to the analysis of expectations and the ways selected by teachers to awaken and maintain students' attention, as well as to recognize their individual differences inside and outside the classroom. The information was obtained through interviews conducted at the Autonomous University of Nuevo León (Mexico). Results and Discussion: Teachers are the ones who make teaching decisions in the classroom, identifying motivation as one of the factors of greater motor skills in the system. Conclusions: Highlights the importance of resignifying educational processes in correspondence with the profiles of the actors.

Keywords: University teaching; decision making; learning expectations; motivation; meaning of teaching 


\section{INTRODUCCIÓN}

La educación universitaria, como expresión del fenómeno educativo, es eminentemente de carácter social, tanto en sus componentes, como en sus contenidos, así como en sus procesos y actores (Durkheim, 1999). Actualmente se presenta como una empresa colectiva, debido a la complejidad de su funcionamiento y requerimientos, por lo que asume un sentido social. Las políticas educativas y económicas, además de las teorías pedagógicas y los materiales educativos prueban que la enseñanza universitaria es una tarea concebida y realizada racional y colectivamente, debido a la planeación que realizan, a los sistemas de calidad que se imponen y a la rendición de cuentas que deben otorgar para demostrar el alcance de sus fines. Por otro lado, los diseños pedagógicos tienden a propiciar el trabajo colaborativo de profesores (Maldonado, 2007) sobre todo en el contexto de la tecnología y la globalización.

En el planteamiento anterior se resalta a la educación como una institución que conjuga las actividades de personas y de organismos, pero de entre todos ellos, resalta la figura del profesor, cuya función se mantiene como fundamental en la enseñanza. Como se puede apreciar, la enseñanza depende de muchos y variados factores, pero finalmente se define en las situaciones de aula a cargo de un profesor, lo cual confiere al trabajo docente su peculiaridad.

El presente artículo aborda el problema de la acción del profesor como agente de la enseñanza, quien debe seguir las directrices institucionales, pero por otro lado debe tomar decisiones acerca de las medidas concretas pertinentes para lograr su propósito. No se desconoce que la enseñanza se enmarca en un medio institucional, en contextos de acción colectiva, pero en este estudio se ubica al profesor en el aula, como responsable directo de su enseñanza, con sus propios medios y recursos profesionales, por más que tenga otros medios institucionales a su disposición.

El objetivo del artículo consiste en analizar las decisiones tomadas por los profesores para adaptar sus acciones de enseñanza de acuerdo con las situaciones cotidianas de aprendizaje y contrastarlas con las expectativas formalmente aceptadas de la enseñanza.

Para identificar las estrategias que sigue el profesor y los elementos que considera para tomar las decisiones se recurrió a entrevistar a profesores reconocidos por sus colegas como "exitosos" cuyas respuestas se revisaron y se organizaron en tres categorías: expectativas del profesor, atención de los estudiantes y lo que ocurre después de la enseñanza en el aula. El concepto de mejores profesores fue elaborado por Bain (2007) en un famoso estudio acerca de lo que hacen estos profesores en su actividad cotidiana, a diferencia de lo que hacen los profesores que siguen las formalidades de enseñanza establecidos por la institución. Sus resultados pusieron en evidencia estrategias de enseñanza relacionadas con saber captar la atención de los estudiantes y mantener la motivación para el aprendizaje dentro y fuera del aula. La posibilidad de tomar decisiones se relaciona con el conocimiento proveniente de la experiencia de estos profesores y sus acciones exitosas son reconocidas tanto por los estudiantes, como por sus colegas.

La presentación del artículo se organiza en atención al orden de los resultados de las entrevistas, los cuales se tratan de interpretar con fundamentos teóricos y experiencias de otras investigaciones similares centradas en la toma de decisiones del profesor en cuanto 
al cambio de las acciones para modificar la efectividad de su enseñanza. La metodología también es expuesta en un apartado, así como la forma de aplicación de entrevistas y su seguimiento. Se agrega un apartado para la discusión y finalmente, se presentan las conclusiones. Los aportes se orientan a puntualizar la centralidad del profesor en el proceso de enseñanza universitaria, lo cual contrasta con los modelos aceptados de centralización de la educación en el estudiante y cuestiona discursos institucionales que ponderan las acciones en busca de calidad, excelencia y competitividad.

\section{Revisión de la literatura}

La enseñanza aborda muchos componentes, pero se concreta finalmente en la actividad de un profesor, por lo que siempre permanece la incertidumbre de la efectividad de los principios educativos institucionales, una vez llevados a la práctica. Inicialmente se puede reducir esta incertidumbre organizando la enseñanza a través de una planificación precisa, lo más pormenorizada posible, integrando todos los elementos, con el propósito de anteponerlos a iniciativas del docente a modo de herramientas de uso obligatorio que aseguren el cumplimiento de las indicaciones institucionales. Aun en el caso de profesores de quienes se dude de su eficacia, tendrían en la planeación de la enseñanza una forma de asegurar que los propósitos educativos se habrán de alcanzar. De acuerdo con esta postura, la enseñanza se concibe como una tarea reductible a reglas provenientes de la investigación educativa aplicada a la enseñanza, esperando que lleguen a buen profesor como un profesionista abocado a la fiel puesta en práctica de la planeación institucional.

Contrariamente a este juicio, la percepción sobre la enseñanza mantiene la resistencia a creer que se pueden confeccionar normas aplicables a todas las situaciones de enseñanza (Maxey, 1956) por depender éstas de la acción personal del profesor en lugar de las herramientas y de la normatividad institucionales. La docencia, en el sentido meramente normativo, se vería reducida a solo una serie de pasos o procedimientos, pero frente a esta postura se contrapone una concepción de la enseñanza como un arte recreado por el profesor, siempre renovado y como tal, dependiente de la reflexión, de la experiencia y de la transformación de las acciones. La enseñanza mejor dirigida, planeada y realizada, no tiene sentido si no provoca el aprendizaje en cada uno de los estudiantes, por lo cual resulta pertinente caracterizar al trabajo docente tanto en lo que le es propio, como en sus semejanzas con otros trabajos.

La docencia comparte con las demás ocupaciones problemas como la explotación y la enajenación, pero también comparte alternativas y desarrollo de atributos, por lo cual se requiere un concepto más amplio del trabajo (Noguera, 2002) en el cual no se vea solo como explotación, sino también como una forma de propiciar el desarrollo individual y social del trabajador. Desde luego que las mejoras laborales difícilmente inciden de manera directa en la efectividad de la docencia, lo cual resulta especialmente importante debido a la dificultad para identificar un producto sobre el cual se pueda apreciar la generación de riqueza obtenida en el proceso (Cornejo, 2006). En este contexto se pueden insertar ciertos indicadores sobre la docencia, con la presunción de que su presencia en el proceso se aseguraría igualmente en el producto. En términos generales, estos atributos son agrupados en el concepto de calidad tanto de la enseñanza, como de la educación. 
La docencia es uno de los campos ocupacionales más concurridos, es desempeñada por profesionistas provenientes de un sinnúmero de orígenes de formación profesional, cuya formación pedagógica poco se jerarquiza, lo cual despierta ciertas suspicacias al momento de analizar la profesionalidad de la docencia universitaria. La docencia es una ocupación compleja debido a problemas en su desempeño (Labaree, 2000). Se pueden identificar fácilmente algunos derivados del crecimiento de la población estudiantil y otros de la situación central que debe asumir el profesor, los cuales agudizan la incertidumbre acerca de la efectividad de la enseñanza, aun sin ser propiamente parte de sus componentes. Con el crecimiento de la matrícula, el profesor debe atender mayor cantidad de estudiantes con perfiles diferentes y con diferentes estrategias de aprendizaje, quienes demandan su atención. En este escenario el profesor debe decidir y actuar por sí mismo, mezclando acciones individuales provenientes de su propia iniciativa, con las prescripciones institucionales, para ser introducidos congruentemente en los procesos de enseñanza y aprendizaje.

Socialmente vista, la posición de la educación superior se ubica en la funcionalidad de la formación profesional y la investigación como proceso de producción de conocimientos y de sus aplicaciones, por lo que pareciera que la enseñanza siempre va atrás o sigue de lejos la dinámica de la sociedad, tratando de entender primero y enseñar después los cambios que ya están ocurriendo, además de pretender atender necesidades sociales y del aparato productivo, aunque ya se hayan acumulado junto a los demás problemas sociales. El profesor primero debe aprender lo que debe enseñar, aun como investigador, por lo cual da la impresión de que su enseñanza es anticuada y el papel que asume es el de cualquier maestro tradicional.

La docencia es una de las ocupaciones más resistente a los cambios, aunque sus agentes, beneficiarios y la propia institución se esfuercen en presentarse como actualizados y hasta innovadores en todos los campos del conocimiento. La calificación de tradicionalista para un profesor no puede ser admitida actualmente en la educación superior, pero en la cotidianeidad de la docencia, las prácticas tradicionales, a diferencia de los contenidos, no son fáciles de sustituir. El profesor mantiene las prácticas que le han resultado efectivas durante su trabajo (Badley y Habeshaw, 1991) se aferra a lo que él y otros profesores, siempre han hecho y con lo que se siente identificado, toma decisiones y dirige las acciones de los estudiantes; su discurso se apega a los fundamentos de la educación, al cumplimiento del deber y al respeto por lo que hace. Por otro lado, el profesor innovador se apropia rápidamente de los nuevos discursos tanto científicos como pedagógicos, además de adoptar las nuevas tecnologías, trata de aplicar los principios que le transmitieron en su formación pedagógica y aspira al reconocimiento y a las recompensas de su esfuerzo, poco valora la tradición y suele desconocer la historia de la institución debido a la inmediatez de las exigencias demandantes de resultados.

La universidad es una institución en la que convergen las tradiciones de la corporación autónoma de especialistas, pero impregnada con las tendencias a la modernidad abocadas hacia la investigación científica con orientación hacia la tecnología aplicada, situadas en las exigencias de la calidad que debe tener toda empresa en la actualidad. Los antecedentes de la enseñanza no desaparecen con el tiempo, sino que permanecen, aunque de manera tenue. La corporación, tanto de profesores como de estudiantes es una de las permanencias más notables de la vida universitaria (Villa, 2017) lo cual es básico para sustentar el estatus 
de autonomía y los derechos de estudiantes y profesores. Sobre esta base se establece la vocación universitaria en torno a la investigación y consecuentemente la libertad de cátedra para poder enseñar.

Los poderes políticos y económicos ejercen fuertes influencias sobre la educación superior, a la vez que la institución se tiene que sustentar, cada vez más, sobre las acciones de los profesores. Los fines institucionales deben ser alcanzados por los profesores; en caso de no lograrlos, por la lógica de la institución, se adjudicaría este fracaso a las acciones no efectivas de los profesores o de los estudiantes, quienes, debido a este esquema, pueden ser rechazados. Estas acciones son parte de las políticas institucionales y gubernamentales, las cuales claramente se sobreponen a las posibilidades de acción de los profesores.

\section{Metodología}

El presente estudio es de tipo descriptivo y se centra en la enseñanza que realiza el profesor universitario como agente activo del proceso escolar, por lo que los datos empíricos que se utilizaron para su desarrollo se sustentan en lo que el propio profesor expresa por ser el informante clave de la enseñanza universitaria. Para alcanzar este acercamiento con los profesores, se utilizó la entrevista a profundidad, la cual con acuerdo a Robles (2011) permite establecer un acercamiento entre iguales a través de conversaciones reiteradas que parecen no seguir un orden determinado, en su lugar se aprovecha cualquier motivo para conversar, el cual se puede dejar si surge algo mejor, se vuelve a algún asunto anterior o, incluso se puede posponer la entrevista.

La entrevista a profundidad permite obtener información directa de los profesores universitarios, proporcionando la materia prima base del conocimiento empírico, pero sus expresiones no se pueden transferir, sin mayor tratamiento al conocimiento del tema. Como toda información, las expresiones de los profesores tienen que ser interpretadas a través de triangulaciones realizadas con la información que ya se tiene, con datos del contexto y, sobre todo, por la reformulación constante de preguntas, aclaraciones y dudas (Coffey y Atkinson, 2003).Una vez obtenidas las declaraciones de los profesores, se procedió a su análisis, primero clasificando cada una en las categorías preestablecidas y posteriormente interpretándolas en su contexto y triangulándolas entre sí, hasta dar congruencia entre interpretaciones con declaraciones. Las categorías que se establecieron fueron tres: expectativas del profesor, formas de motivar a los estudiantes y lo que hacen los estudiantes después de dejar el aula.

La selección de los entrevistados se realizó a través de recomendaciones personales de los propios profesores. Inicialmente se contactó a profesores para preguntarles acerca de su conocimiento de algún colega que fuera reconocido como un "buen profesor" en el sentido de su distinguido desempeño como docente, se aclaró que no se trataba de buscar profesores con elevados logros académicos, de investigación o de difusión, sino que se estaba enfocando en docentes que cumplieran a cabalidad con esta función, que lo hicieran con entusiasmo y con entrega permanentes. De este modo se logró localizar a los ocho profesores, quienes aceptaron la entrevista y solo en un caso, la sesión fue breve, debido a las ocupaciones del profesor. El procedimiento de selección probablemente provocó que entre los entrevistados no hubiera ningún profesor que ocupara un puesto directivo y sobre todo, resalta el hecho de 
su elevada antigüedad mayor a los treinta años, puesto que para obtener el reconocimiento de sus colegas, se requiere haber sido profesor por un prolongado tiempo.

Las entrevistas fueron aplicadas a los profesores durante el transcurso de varios meses del año 2019 y tuvieron duración variable, desde los 20 minutos hasta más de dos horas, además casi todas se extendieron a varias sesiones en días diferentes. El lugar y la fecha de la entrevista fueron acordadas previamente con cada uno de los ocho profesores de manera individual. La totalidad de las entrevistas se realizaron dentro de las instalaciones de la Universidad Autónoma de Nuevo León, en los cubículos de trabajo de los profesores, dos ocasiones en consultorio médico, una en laboratorio y otra en la misma aula, antes de la entrada de los estudiantes. Los contenidos de las entrevistas fueron registrados en notas escritas tomadas por el propio entrevistador y transcritas completas una vez terminada la sesión, en ningún caso se utilizó aparato para grabar de voz ni video.

\section{RESUltados}

Para su organización, los resultados se clasificaron en tres categorías conforme a los momentos en los que el profesor debe tomar las decisiones que considerara pertinentes, tomando en cuenta el cambio de contextos. La primera categoría corresponde a las expectativas que tiene el profesor antes de iniciar su sesión de aula. La segunda corresponde a los inicios de la sesión, después de replantear las expectativas previas y la tercera se ubica una vez que se termina la sesión y se pueden plantear nuevas expectativas para las sesiones posteriores. Las categorías se encuentran íntimamente interconectadas y son fundamentales para la determinación de las acciones docentes desde la posición del profesor, sobrepasando los lineamientos institucionales. Las categorías y su contenido se presentan en la Tabla 1. Previamente a los resultados se presentan los datos generales de los profesores entrevistados.

TABLA 1.

Categorías de análisis.

\begin{tabular}{ll}
\hline \multicolumn{1}{c}{ Categoría } & \multicolumn{1}{c}{ Contenido } \\
\hline Expectativas del profesor. & Qué espera el profesor de sus estudiantes, con base en su experiencia. \\
Motivación. & Formas para despertar y mantener la atención de los estudiantes. \\
Enseñanza después del aula. & Lo que hacen los profesores y los estudiantes después de la enseñanza en el aula. \\
\hline
\end{tabular}

Nota: Esta tabla presentan las categorías sobre la toma de decisión del profesor para realizar la enseñanza. Fuente: Elaboración propia.

Las categorías enunciadas en la Tabla 1, se aprecian las diversas situaciones en las cuales el profesor debe tomar decisiones sobre la enseñanza. En la primera categoría se hace alusión a lo esperado por el profesor, antes de iniciar su clase, sin conocer todavía las características de sus estudiantes ni los contextos. Una vez iniciada la sesión de clase y replanteadas las expectativas iniciales, el profesor decide como conducir su clase, recurriendo regularmente a estrategias de motivación debido a los nuevos contextos. En la categoría tres, el profesor reflexiona sobre nuevas estrategias, una vez que la sesión de clase ha terminado. 


\section{Datos generales}

Los profesores entrevistados suman un total de ocho, cinco del género masculino y tres del femenino. Todos ellos se encontraban laborando en la Universidad Autónoma de Nuevo León (México) al momento de hacer la entrevista, desempeñándose como docentes ya sea en licenciatura, en posgrado o en ambos niveles y cuentan con una antigüedad laboral por encima de los 30 años en promedio. Todos los profesores cuentan con posgrado, seis de doctorado y dos de maestría, en sus áreas de dominio profesional. Los campos disciplinares en los que ejercen su docencia son los de ciencias de la salud, ciencias exactas, ingeniería y un profesor de humanidades. Los datos más relevantes de este apartado se presentan en la siguiente Tabla 2.

TABLA 2.

Áreas de desempeño de los docentes.

\begin{tabular}{lll}
\hline \multicolumn{1}{c}{ Área de desempeño } & Número de profesores \\
\hline Ciencias de la salud & 3 \\
Ciencias Exactas & 2 \\
Ingeniería & 2 \\
Humanidades & 1 \\
Total & 8 \\
\hline
\end{tabular}

Nota: Esta tabla se presentan las áreas de desempeño de docencia de los profesores, iniciando con la de mayor frecuencia, y sumando el total de los ocho profesores entrevistados. Fuente: Elaboración propia.

En la Tabla 2 se puede apreciar la variedad de áreas en las donde los profesores ejercen su docencia. El área predominante es el de las ciencias de la salud, seguida por el área de ingeniería y ciencias exactas. Es notoria la escasa presencia de profesores del área de humanidades y la ausencia de ciencias sociales, pudiendo provocar cierto sesgo.

Los resultados se presentan en los siguientes subtemas organizados con acuerdo a las categorías identificadas en las entrevistas con los profesores.

\section{Expectativas de Aprendizaje}

El sentido más valioso de la enseñanza es sin duda el de provocar el aprendizaje de los estudiantes, lo cual provoca en los profesores la construcción de expectativas en cuanto los alcances de su enseñanza en relación con al aprendizaje. La importancia de las expectativas radica en su influencia en la toma de decisiones de los profesores (Valle y Nuñez, 1989). Las expectativas interactúan con otros contextos del aula y provocan nuevas situaciones de aprendizaje.

Las expectativas usuales del profesor se refieren a los métodos, contenidos y logros del proceso de enseñanza, los cuales toman relieve sobre todo al inicio de cada curso, cuando no se conoce aún a los estudiantes. En este momento se configuran las expectativas dependiendo de la experiencia del profesor, de la trayectoria de los estudiantes y de las políticas institucionales, pero en este estudio las expectativas no se refieren a los planos formales de la enseñanza sino más bien a lo que los profesores pueden establecer por sí mismos. 
Para averiguar las expectativas del profesor se plantearon preguntas acerca de: expectativas acerca del aprendizaje de sus estudiantes, cumplimiento de actividades, superación de problemas de aprendizaje, del pensamiento crítico, diferencias individuales y significado del aprendizaje, los cuales se describen a continuación.

Aprendizaje de contenidos y cumplimiento de actividades. De acuerdo con la información proporcionada, los profesores no esperan de sus estudiantes el aprendizaje de "muchos" contenidos, ni siquiera la mayoría de los que se propone enseñar, tampoco esperan el cumplimiento de la totalidad de las actividades diseñadas, a lo sumo, como uno de ellos lo expresó, lo espera es "que sean responsables, que cumplan con su deber, con lo que se espera de ellos". En un grupo de estudiantes, aunque formalmente todos ellos sean iguales, son siempre diferentes, en menor o mayor medida se congrega diferentes orígenes, trayectorias y estilos personales para aprender. Cuando un profesor se dirige a ellos lo hace en colectivo, pero sabe de sus diferencias, por lo cual espera resultados diferentes.

La apropiación de todos los contenidos enseñados no es una expectativa realista, por lo que el profesor no se la plantea, en su lugar espera lograr objetivos más pertinentes, sobrepasando los marcos de los conocimientos para llegar a los planos actitudinales como el de la responsabilidad y el cumplimiento del deber. La realización de las actividades por parte de los estudiantes que el profesor establece en su planeación tampoco es garantía de aprovechamiento en el aprendizaje, pero sí son garantía de cumplimiento con el deber, por lo que es deseable que se realicen como prácticas ya sean individuales o en forma colectiva.

Superar problemas. El profesor sabe que los estudiantes no tienen solo una vida escolar, pues viven diversas situaciones personales, sociales, familiares, que muchos de ellos tienen problemas económicos que interfieren con su aprovechamiento escolar además de vivir muchas otras situaciones de diverso tipo. La vida personal de los estudiantes no puede despertar muchas expectativas, pues los profesores saben que está fuera de su marco de acción, cuando mucho conceden que los estudiantes pasan por muy diversas y problemáticas situaciones, pero que no pueden hacer gran cosa frente a ellas, a lo sumo se dedican a dar consejos sobre los hábitos de salud, y a dictar discursos acerca de la superación de los obstáculos para resolver los problemas. Es de resaltar que ninguno de los profesores habló de las tutorías, a pesar de ser una actividad que obligatoriamente realizan y en las que se pueden tratar los asuntos relacionados con los problemas del estudiante.

Pensamiento crítico. Éste es uno de los atributos más importantes que se busca lograr con la educación superior, pero no obstante su aceptación, es difícil localizar una definición (Vero y Puka, 2018) que exprese claramente el contenido de esta cualidad, pues abarca una serie de características complejas y más difícil resulta, conjugar esas características con las finalidades institucionales de la educación. Mención aparte merece la idea de llevar el pensamiento crítico a las situaciones cotidianas de aula (López, 2012) en el contexto de la enseñanza actual. Los comentarios de los profesores sobre este aspecto fueron solo superficiales, aceptaban que con la enseñanza pretendían promover el desarrollo del pensamiento crítico, pero sin definir lo que querían decir ni como lo apropiarían los estudiantes. Tampoco hicieron referencia a la forma como lo integran a la enseñanza ni como podría ser evaluado, por lo que no se puede aceptar que tengan una expectativa clara en este aspecto. 
Diferencias Individuales. El trabajo docente se desempeña generalmente atendiendo grupos de estudiantes, y con menos frecuencia se trabaja con alguien en lo particular, pero los profesores reconocen que las diferencias entre sus estudiantes se vuelven significativas para su aprendizaje, por lo que no se puede exigir a todos los estudiantes el mismo rendimiento académico. Si bien la institución reglamenta los dominios de aprendizaje necesarios para la promoción de grado escolar, el profesor por su parte mantiene el poder de la decisión de asignar las notas de evaluación del estudiante. Las notas de evaluación son evidentemente diferentes al aprovechamiento escolar, aunque pretendan ser lo mismo; por otro lado, el profesor tiene control de las notas, aunque no del aprendizaje, lo que le concede un lugar estratégico en la relación con los estudiantes, pero que es potencialmente conflictivo con la institución. Los profesores logran articular sus evaluaciones entre los reglamentos de la institución y las expectativas de los estudiantes, por lo cual establecen sus propios parámetros, sin contradecir explícitamente los reglamentos y sin decepcionar a los estudiantes.

Las diferencias individuales no provocan que los profesores cambien su forma de enseñanza, aunque si bien las reconocen, siguen actuando como si el grupo fuera homogéneo; a lo sumo, cuando explican algún asunto preguntan al grupo si alguien tiene alguna duda o que si todos han entendido su exposición; por supuesto que casi nunca hay respuestas de los estudiantes, tengan o no tengan dudas o hayan o no entendido las explicaciones. El profesor cumple con sus deberes, aunque no verifique el aprendizaje y menos la individualidad de los estudiantes, pero puede continuar con su enseñanza, hasta el momento cuando deba evaluar el aprendizaje, sin saber de la efectividad de su trabajo, pero seguro de que cualquier problema lo podrá resolver a través de la asignación de las notas de evaluación, con las que podrá compensar las deficiencias del proceso y equilibrar las expectativas de la institución.

Significado del Aprendizaje. Los estudiantes de bajo aprovechamiento son los que suelen demandar más esfuerzos al profesor debido a que se les tiene que prestar atención especial y apoyar de diversas maneras en su aprendizaje, mientras que los estudiantes brillantes pasan desapercibidos sobre todo porque suelen cumplir fácilmente las tareas, desaprovechando el potencial que pudieran tener.

Si bien la institución escolar tiene fines específicos con respecto a la formación de profesionistas, en la cotidianeidad del aula los fines aparecen demasiado lejanos para la realidad de la enseñanza, además de que el profesor no tiene vinculación directa con el futuro desempeño profesional de sus estudiantes. La enseñanza es mucho más concreta que los fines institucionales, por lo que, se acepta de buen grado que los fines formativos para el desarrollo humano, cívicos y éticos de los estudiantes sea una finalidad, no solo en el discurso, sino dentro de las acciones que se verifican en el aula. Si un estudiante no logra los niveles mínimos de aprovechamiento académico, no por eso se le va a negar la promoción si ha tratado de cumplirlos con base en esfuerzo, aunque no sea muy productivo. Algunos profesores expresaron su admiración al percatarse que profesionistas destacados habían sido estudiantes que apenas cumplían con sus tareas, aunque reconocieron que evidentemente tenían otras aptitudes e intereses, por lo que se congratulaban de no haberlos reprobado. 


\section{Motivación}

Las expectativas son una especie de metas que el profesor reconoce como posibles de lograr con base en su experiencia dentro del contexto del cumplimiento de su compromiso con la institución. En el desempeño del trabajo docente el profesor, en la independencia que le otorga el aula, hace uso discrecional de todos los recursos y mecanismos: tiempo, materiales, espacio y evaluaciones por lo que está en posibilidad de decidir sus acciones, sin más presión que el cumplimiento formal de su deber. El inicio de cada sesión de clase, aunque parezca algo habitual, se convierte en toda una incertidumbre. No nos referimos a los inicios habituales de cada sesión, sino a las interrogantes que el profesor se plantea como dudas e inquietudes sobre lo que puede hacer como docente al tener que iniciar cada sesión de clase. En este sentido se les cuestionó a los profesores acerca de: los modos como inician las clases, motivaciones que utilizan y formas para lograr y mantener la atención (Tabla 3).

TABLA 3.

Expectativas del profesor.

\begin{tabular}{|c|c|c|c|}
\hline $\begin{array}{l}\text { Aspectos sobre los que se } \\
\text { establecen las expectativas } \\
\text { del aprendizaje }\end{array}$ & $\begin{array}{c}\text { Postulado } \\
\text { institucional }\end{array}$ & Lo no esperado & Lo realmente esperado \\
\hline Contenidos. & $\begin{array}{l}\text { Los estudiantes deben } \\
\text { apropiarse de todos o } \\
\text { la mayor parte de los } \\
\text { contenidos. }\end{array}$ & $\begin{array}{l}\text { No se espera que todos } \\
\text { los estudiantes apropien } \\
\text { todos los contenidos. }\end{array}$ & $\begin{array}{l}\text { Esperan que los } \\
\text { estudiantes se esfuercen, } \\
\text { que se responsabilicen de } \\
\text { su trabajo. }\end{array}$ \\
\hline Actividades. & $\begin{array}{l}\text { Los estudiantes deben } \\
\text { realizar todas o la } \\
\text { mayor parte de las } \\
\text { actividades. }\end{array}$ & $\begin{array}{l}\text { No se espera que todos } \\
\text { los estudiantes realicen } \\
\text { todas las actividades. }\end{array}$ & $\begin{array}{l}\text { Esperan que los } \\
\text { estudiantes se esfuercen, } \\
\text { que se responsabilicen de } \\
\text { su trabajo. }\end{array}$ \\
\hline $\begin{array}{l}\text { Problemas y situaciones } \\
\text { de los estudiantes. }\end{array}$ & $\begin{array}{l}\text { Los estudiantes son } \\
\text { iguales en lo general. }\end{array}$ & $\begin{array}{l}\text { No se espera que los } \\
\text { estudiantes se deshagan } \\
\text { de sus problemas. }\end{array}$ & $\begin{array}{l}\text { Se espera que puedan } \\
\text { cumplir con su trabajo, } \\
\text { anteponiéndose a sus } \\
\text { problemas. }\end{array}$ \\
\hline Pensamiento crítico. & $\begin{array}{l}\text { Los estudiantes } \\
\text { deben desarrollar el } \\
\text { pensamiento crítico. }\end{array}$ & $\begin{array}{l}\text { No se espera que los } \\
\text { estudiantes sean críticos. }\end{array}$ & $\begin{array}{l}\text { Que los estudiantes } \\
\text { cumplan con su deber. }\end{array}$ \\
\hline $\begin{array}{l}\text { Diferencias individuales de } \\
\text { los estudiantes. }\end{array}$ & $\begin{array}{l}\text { Las diferencias se } \\
\text { deben establecer por } \\
\text { los méritos de cada } \\
\text { estudiante. }\end{array}$ & $\begin{array}{l}\text { No se espera que } \\
\text { automáticamente los } \\
\text { "estudiantes exitosos" } \\
\text { se conviertan en } \\
\text { "profesionistas exitosos". }\end{array}$ & $\begin{array}{l}\text { Que los estudiantes } \\
\text { terminen sus estudios y } \\
\text { encuentren empleo. }\end{array}$ \\
\hline $\begin{array}{l}\text { Significado del } \\
\text { aprendizaje. }\end{array}$ & $\begin{array}{l}\text { Apropiación de } \\
\text { contenidos por parte de } \\
\text { los estudiantes. }\end{array}$ & $\begin{array}{l}\text { No se espera que los } \\
\text { estudiantes valoren } \\
\text { cabalmente los } \\
\text { conocimientos que le } \\
\text { enseñan. }\end{array}$ & $\begin{array}{l}\text { Que los estudiantes } \\
\text { aprecien algo de la labor } \\
\text { del profesor. }\end{array}$ \\
\hline
\end{tabular}

Nota: en las columnas de esta tabla se puede observar las diferencias entre las expectativas del profesor en diferentes ámbitos de la docencia. La primera columna se integra con las expectativas establecidas formalmente a nivel institucional, la segunda contiene las expectativas consideradas como poco alcanzables por la generalidad de los estudiantes, mientras que la tercera hace referencia a expectativas realistas para con los estudiantes. Las filas de la tabla se confeccionaron siguiendo las situaciones que los profesores resaltaban en sus intervenciones como importantes para la toma de decisiones, como el contenido de la enseñanza, las actividades de aprendizaje, las diferencias individuales, el pensamiento crítico y el significado del aprendizaje. Fuente: Elaboración propia. 
Inicio de clases. El inicio de la clase es fundamental para su desarrollo, por lo que no es de extrañar que se resalte el hecho de que, para iniciar su clase, los profesores se valen de recursos variados, obtenidos ocasionalmente de diversas fuentes como las noticias del día, tendencias en la red, los deportes, algún personaje, aunque muchas veces, estos comentarios no tengan nada que ver con los contenidos formales. No se considera que esto sea pérdida de tiempo, ya que se gana mucho despertando la simpatía hacia la persona del profesor y el interés acerca de temas relevantes. Incluso, no faltan profesores que inician la clase hablando de sus situaciones personales o familiares, tratando de ser identificados como personas ordinarias y no solo como profesionales de la academia. De una u otra manera, se puede decir que esto que hace el profesor, es una especie de motivación, nada estructurada, pero sí efectiva dentro de la cotidianeidad, asumiendo la postura de que la motivación es algo sumamente complejo (Polanco, 2005) en su relación con el aprendizaje, sobre todo en los niveles de educación superior.

Formas de motivación. Otras formas de motivación, aunque no sean positivas, son las amenazas y castigos que se mencionaron por los profesores, a la manea de advertencias como "leer la cartilla", "poner las cosas en claro" y exhortaciones para no sufrir las consecuencias del incumplimiento de las actividades. Los halagos, buenos deseos y palabras de aliento tampoco estuvieron ausentes para motivar el aprendizaje. Los llamados a la competencia para "ser el mejor" "un triunfador" y otros semejantes no se mencionaron, aunque es posible que se utilicen. Por otro lado, los llamados "desafíos intelectuales" (Bain, 2007) les parecieron interesantes y atractivos a los profesores, pero ninguno de ellos afirmó que los utilizaba a pesar de su enfoque académico y profesional. Las motivaciones se centran mayormente en mejorar el ánimo de los estudiantes, despertar su interés, llamar su atención, es decir, se enfocan en el estudiante, por lo que las aventuras intelectuales, retos o desafíos científicos o de dominio de conocimientos no son calificadas como motivantes, sino simplemente como lo que el profesor debe cumplir.

El supuesto sobre el que se sustenta la motivación es que incidirá positivamente en el aprendizaje del estudiante, debiéndose reflejar finalmente en sus evaluaciones. Las menciones sobre este asunto no se expresaron por parte de los profesores, sin poder determinar si es simplemente porque no lo hacen o si no lo consideran relevante. De todos modos, se considera que la motivación es algo deseable por lo menos para provocar un buen ambiente en la relación del profesor con los estudiantes. Es de suponerse también que los profesores que motivan a sus estudiantes se preocupan mayormente por la buena conducción de su enseñanza, que aquellos profesores que no lo hacen. Permanece igualmente la controversia sobre la necesidad de motivar a los estudiantes en la educación superior debido a la presunción de su interés ya establecido por su condición de adultos, de convertirse en profesionistas y por la interacción con el medio social y familiar propicio para cursar una carrera universitaria.

La motivación debe ser permanente parte fundamental de la misma enseñanza y no solo recurrir a ella al inicio de cada sesión de los cursos, pero aunque se presente solo esporádicamente, siempre es bien vista, a pesar de que por otro lado, es fácil deducir que la motivación no puede constituir por sí misma toda la enseñanza, puesto que es un medio para despertar el aprendizaje y de ningún modo se puede convertir en su sustituto. El análisis de la enseñanza nos conduce a destacar la interacción del profesor con los estudiantes (Covarrubias y Piña, 2004) como uno de los contextos fundamentales de la educación superior. Para indagar acerca de esta temática se hicieron preguntas referentes a: ¿cómo logra despertar y mantener la 
atención de los estudiantes, una vez iniciada la clase?, ¿cómo se percata de que sus estudiantes le prestan la atención debida?, ¿se pierde la atención en algún momento de la clase?, ¿cómo le hace para recuperarla?

Atención. El profesor es quien realmente conduce la enseñanza y el responsable directo de este proceso, los materiales y apoyos educativos no pueden ubicarse sobre su papel. Las condiciones de trabajo docente se centran en el profesor, incrementando presiones sobre el cumplimiento de las metas establecidas por la institución, asignadas a él. El número de estudiantes es un factor que incide en las presiones al demandar atención individual a cada uno de ellos, al igual que tener que ir de un grupo a otro para impartir una clase, o atender a un estudiante tras otro formados en una lista de espera, hace que la atención brindada sea lo más rápida posible, dejando de lado muchas situaciones que pudieran ser importantes.

La importancia de despertar y mantener la atención de todos los estudiantes en los diversos grupos, es ampliamente reconocida, por lo que se han diseñado recomendaciones didácticas para este propósito dentro del conjunto de actividades de la clase del profesor a través de sus exposiciones orales (Del Barrio y Borragán, 2011). Los profesores entrevistados conceden la importancia debida al mantenimiento de la atención de los estudiantes y deben realizar grandes esfuerzos por lograrlo. Las formas que mencionaron para conseguirlo son más bien recursos ocasionales como pedir a los estudiantes que se pongan de pie para que muevan un poco su cuerpo, que los estudiantes que ocupan los lugares del fondo del aula se cambien a las primeras filas. Otros recursos son más propios del profesor como moverse él mismo por toda la superficie del aula, levantar la voz, dirigirse a un estudiante en lo particular y hasta interrogar directamente a los estudiantes acerca de sus exposiciones. Todas estas acciones las realiza el profesor porque quiere recuperar o mantener la atención de los estudiantes, una vez que ha menguado el impulso inicial de la clase. Las situaciones en las donde el profesor decide motivar a sus estudiantes, así como las formas y el significado de la motivación se presentan a continuación organizadas en la Tabla 4.

TABLA 4.

Formas de motivar y mantener la atención de los estudiantes.

\begin{tabular}{llll}
\hline $\begin{array}{c}\text { Situaciones en las } \\
\text { que se establece } \\
\text { la motivación }\end{array}$ & \multicolumn{1}{c}{$\begin{array}{c}\text { Postulado } \\
\text { institucional }\end{array}$} & \multicolumn{1}{c}{ Formas de motivación } & Significado de la motivación \\
\hline $\begin{array}{l}\text { Al inicio de cada } \\
\text { clase. }\end{array}$ & $\begin{array}{l}\text { Los estudiantes } \\
\text { deben llegar a la } \\
\text { clase motivados. }\end{array}$ & $\begin{array}{l}\text { Inicia la clase hablando de } \\
\text { noticias, deportes, situaciones } \\
\text { familiares, personajes, } \\
\text { tendencias, experiencias, etc. }\end{array}$ & $\begin{array}{l}\text { Despierta la confianza, } \\
\text { simpatía, cotidianeidad. } \\
\text { No es pérdida de tiempo. }\end{array}$ \\
Durante la clase. & $\begin{array}{l}\text { Los estudiantes } \\
\text { deben permanecer } \\
\text { motivados. }\end{array}$ & $\begin{array}{l}\text { Desafíos intelectuales y } \\
\text { personales, experiencias, } \\
\text { preguntas directas. }\end{array}$ & $\begin{array}{l}\text { Se requiere recuperar la } \\
\text { atención de los estudiantes, } \\
\text { que fácilmente se pierde. }\end{array}$ \\
$\begin{array}{l}\text { Mantenimiento de la } \\
\text { atención. }\end{array}$ & $\begin{array}{l}\text { Desplazarse a lo largo del aula, } \\
\text { deben estar } \\
\text { atentos. }\end{array}$ & $\begin{array}{l}\text { Lambiar lugares, alternar } \\
\text { actividades. }\end{array}$ & $\begin{array}{l}\text { mantienen la atención } \\
\text { durante mucho tiempo. }\end{array}$ \\
\hline
\end{tabular}

Nota: Las dos últimas columnas de la Tabla 4 presentan las diversas formas de motivación utilizadas por el profesor y su significado con relación a los problemas detectados, las columnas se pueden contrastar con los supuestos institucionales o formales. Las filas se confeccionaron con atención a los momentos o situaciones del desarrollo de la clase, debido a la dinámica del desarrollo de la enseñanza. Como se puede apreciar en las casillas de la tabla, las estrategias de motivación tratan de responder a los momentos y situaciones de enseñanza. Fuente: Elaboración propia. 
La situación y los contextos de enseñanza en el aula contienen una dinámica propia, ante la cual, el profesor debe variar sus formas de motivación tratando de mantener la atención de los estudiantes, pero sobre todo, se pude destacar su significado en el contexto de la experiencia del profesor.

\section{Antes y después del Aula}

El aula, como espacio dedicado a la enseñanza y el aprendizaje, se reconoce por sus límites, tanto espaciales como temporales, pero sobre todo el aula se define porque sintetiza los procesos que supone se deben realizar previamente, por parte de los profesores aparece la planeación didáctica, mientras que por parte de los estudiantes, el cumplimiento de tareas asignadas por el profesor, la enseñanza, por tanto se compone de actividades antes y después de los encuentros en el aula, por lo que es necesario analizarlos.

Finalización de clase. La planeación del desarrollo de una sesión habitual de clase presencial prevé también la forma cómo habrá de finalizar, puesto que este último momento es crucial para retomar la enseñanza en las sesiones posteriores. Obviamente que el tiempo de duración de las sesiones de clase se encuentra predeterminado por un horario y que son múltiples las circunstancias que hacen variar los tiempos de aprovechamiento para el aprendizaje, pero ubicados en este contexto, terminar una sesión es mucho más problemático que simplemente agotar el tiempo disponible, puesto que dar por terminada una clase no es lo mismo que agotar el tiempo, debido a que un buen cierre corresponde específicamente a la planeación (Cáceres y Rivera, 2017) de la sesión de clase.

Al interrogar a los profesores específicamente a través de cuestionamientos como: ¿cómo concluye sus clases?, ¿resuelve los problemas planteados?, ¿llega a conclusiones?, ¿dice que continuará la próxima clase?, ¿simplemente la da por terminada?, se expresaron un tanto sorprendidos de la pregunta, debido principalmente a que suponen que la planeación de la clase se hace para todo el tiempo programado por lo que no hay necesidad de planear algo específico para este momento. En este sentido un profesor mencionó que "se termina de acuerdo con la planeación" otros profesores comprenden que la finalización de la clase es un momento crucial tanto para dar por concluida la sesión, como para poder iniciar, en un tiempo posterior, otra nueva. Estos profesores establecen actividades de cierre diciendo: "termino con un mini examen sobre los aspectos más importantes de la clase" el cual no está impreso, sino que se contesta en una hoja de libreta o que consiste en un interrogatorio al grupo acerca de lo que el profesor considera que es lo más importante.

La terminación de una sesión de clase significa que el proceso de enseñanza sigue abierto para una serie de actividades posibles fuera del aula y del edificio escolar. La institución universitaria puede ofrecer espacios e instalaciones adecuadas que satisfagan todas las necesidades para la realización de las actividades de aprendizaje, además de las aulas, por lo que el estudiante debe pasar ese tiempo en la universidad realizando las actividades de aula y extra-aula. Eventualmente, algunos trabajos se deben realizar fuera del edificio, sobre todo cuando son de carácter práctico en el campo profesional. 
Actividades extra-aula. El problema principal de las actividades extra-aula es que deben mantener el mismo carácter activo de las realizadas en el aula (Prince, 2004) pero sin la presencia del profesor, por lo que no cuentan con controles ni asesorías, sino que se tienen que sustentar sobre los propios hábitos de aprendizaje de los estudiantes, incidiendo en los campos del auto aprendizaje. La planeación pormenorizada de la enseñanza prevé la realización de actividades por parte de los estudiantes tanto en forma individual como colectiva, así como las que se efectúan dentro y fuera del aula, lo cual no significa que el profesor pueda verificar el desempeño de los estudiantes, sin embargo, tiene que validarlo de una u otra forma. La asignación de tareas es algo común por parte de los profesores, puesto que se considera que el tiempo de aula siempre es insuficiente para alcanzar los fines de la enseñanza.

Las razones para recurrir a actividades fuera del aula se fundamentan, además de la escasez del tiempo, en que son diferentes y que se deben realizar más allá de los espacios escolares, son actividades tanto de preparación para el aula como para continuar con los pendientes. La mayor distinción que pueden tener estas actividades es que realmente significan aprendizajes diferentes a los del aula que enriquecen la formación del estudiante. De ninguna manera se aceptaría que fuera del aula se repitiera lo que ya se ha hecho dentro, solo para mantener en actividad al estudiante (Tabla 5).

TABLA 5.

Fuera del aula.

\begin{tabular}{llll}
\hline Finalización de la clase & Postulado institucional & \multicolumn{1}{c}{$\begin{array}{c}\text { Formas de } \\
\text { terminación }\end{array}$} & $\begin{array}{c}\text { Significado de la } \\
\text { terminación }\end{array}$ \\
\hline Al terminar el tiempo. & $\begin{array}{l}\text { Los estudiantes deben } \\
\text { realizar actividades } \\
\text { todo el tiempo. }\end{array}$ & $\begin{array}{l}\text { Repaso general } \\
\text { Evaluación breve } \\
\text { Asignación de tareas. }\end{array}$ & $\begin{array}{l}\text { Continuidad de la } \\
\text { enseñanza. }\end{array}$ \\
& $\begin{array}{l}\text { Los estudiantes deben } \\
\text { realizar actividades } \\
\text { después del tiempo de } \\
\text { aula. }\end{array}$ & $\begin{array}{l}\text { Reafirmar que se } \\
\text { deben realizar más } \\
\text { actividades para la } \\
\text { próxima clase. }\end{array}$ & $\begin{array}{l}\text { Planeación de la } \\
\text { siguiente clase. }\end{array}$ \\
\hline
\end{tabular}

Nota: La finalización de la clase se considera igual de importante a los otros momentos, pero tiene un especial significado debido a que, supuestamente no se puede dar por terminado y ni siquiera pospuesto ningún proceso de enseñanza, sin antes verificar el cumplimiento de los propósitos en el aprendizaje, sin embargo, la terminación de la clase se puede verificar de diversas formas, incluyendo la simple terminación del tiempo asignado, por ello, el cierre de las clases tiene igualmente un significado desde la experiencia del profesor. Fuente: Elaboración propia.

\section{DiscUSIÓN}

Los resultados del presente estudio permiten afirmar que los profesores mantienen el papel central de la enseñanza en el aula quienes deben tomar decisiones para cumplir con su rol, interactuando con los estudiantes y desenvolviéndose en el contexto institu- 
cional. Las decisiones tomadas por el profesor tienen como antecedente el conocimiento previo adquirido durante su experiencia, asumiendo que buena parte de sus acciones se deciden bajo este supuesto, las cuales adquieren relieve sobre todo al comenzar un nuevo curso.

Las categorías establecidas permitieron analizar importantes aspectos de la docencia, comenzando por las expectativas generadas por los profesores, dentro de ellas, se destaca, la expectativa de que sus estudiantes adopten las actitudes de responsabilidad para cumplir con su deber para ser considerados buenos estudiantes. Frente a desafíos como el de los problemas y las diferencias individuales que tienen los estudiantes, los profesores aceptan las dificultades para poder incidir positivamente en estos problemas, y decididamente los toman en cuenta al momento de enseñar. Como una muestra del cumplimiento de los profesores, se menciona el desarrollo del pensamiento crítico, el cual, a pesar de no poder definirlo, afirma que sí lo promueven. En general los profesores cumplen con las directrices institucionales, pero sus expectativas para con los estudiantes reflejan fines formativos y aceptan sus limitadas posibilidades de interceder en los problemas y diferencias entre ellos. Las expectativas orientan las acciones posteriores de los profesores, pero solo si se centran en fines formativos es de esperarse que sus acciones sigan esta dirección, por lo que atraer el interés de los estudiantes resulta fundamental para despertar y mantener la motivación, no necesariamente de contenidos académicos sino de actitudes consideradas de mayor relieve educativo.

Las acciones del profesor son tomadas individualmente y con posibilidades de aplicar, gracias a que ni los condicionamientos institucionales ni las políticas globales pueden llegar hasta el aula, en donde el profesor sigue ejerciendo la exclusividad de la conducción de la enseñanza.

\section{CONCLUSIONES}

En correspondencia con los objetivos del artículo se concluye que:

El proceso de enseñanza se centra en el profesor, quien está en posición de tomar las decisiones pertinentes para modificar sus acciones con acuerdo en la efectividad en el aprendizaje de los estudiantes.

Las decisiones para modificar la enseñanza se identifican a través de su comparación con las expectativas establecidas en el nivel de planeación formal, una vez que se inicia el proceso de enseñanza. Los momentos significativos para la toma de decisiones se ubicaron al inicio de la clase, durante la clase y después de la sesión. Los cambios en las estrategias de enseñanza se orientaron hacia la búsqueda del mantenimiento de la motivación de los estudiantes durante y después de las sesiones de aula.

Los ámbitos de enseñanza, resaltados por los propios profesores, en los que se toman las decisiones abarcan los contenidos a enseñar, las actividades de los estudiantes, la formación del pensamiento crítico, las acciones frente a las diferencias individuales de los estudiantes y el significado del aprendizaje. En todos ellos, los profesores se enfocaron hacia cambios contextualizados en las condiciones concretas de la enseñanza y de los estudiantes, destacando el planteamiento de los contenidos de enseñanza donde los cambios contrastan notoriamente de las expectativas formales. 
Las formas de motivación fueron las más mencionadas por los profesores, abundando sus formas y procedimientos, todas ellas se orientaron por la intención de mantener el interés de los estudiantes.

Las estrategias de terminación de las clases representaban un problema, debido a las limitaciones establecidas por el horario de la clase y a la falta de alternativas previstas para su solución, sobre todo, derivada del problema de verificar el aprendizaje de los estudiantes.

De especial importancia resulta el cambio de significados de las expectativas establecidas, pues ubican al profesor en el centro de la toma de decisiones de la enseñanza, gracias al conocimiento proveniente de su experiencia y al reconocimiento de estudiantes y de otros profesores, lo cual los faculta para realizar los cambios que considera convenientes.

\section{REFERENCIAS}

Badley, G. y Habeshaw, T. (1991). The Changing Role of the Teacher in Higher Education. Journal of in-Service Education, 17(3), 212-218. https://oi. org/10.1080/0305763910170307

Bain, K. (2007). Lo que hacen los mejores profesores de universidad. Valencia: Universitat de Valencia.

Cáceres, M. y Rivera, P. (2017). El docente universitario y su rol en la planificación de la sesión de enseñanza - aprendizaje. En Blanco y Negro, 8(1), 15-27. Disponible en https://revistas.pucp.edu.pe/index.php/enblancoynegro/article/view/18958

Coffey, A. y Atkinson, P. (2003). Encontrar el sentido de los datos cualitativos. Estrategias complementarias de investigación. Medellín: Universidad de Antioquía.

Cornejo, R. (2006). El trabajo docente en la institución escolar La apropiación-enajenación del proceso de trabajo docente en el contexto de las reformas educativas neoliberales. Revista de Psicología, 15(2), 9-28. https://doi.org/10.5354/07190581.2006 .18390

Covarrubias, P. y Piña, M. M. (2004). La interacción maestro-alumno y su relación con el aprendizaje. Revista Latinoamericana de Estudios Educativos (México), XXXIV(1), 47-84. Disponible en https://rlee.ibero.mx/index.php/rlee/issue/view/141

Del Barrio, J. A. y Borragán, A. (2014). Cómo atraer la atención hablando. Un reto para la enseñanza. Bordón. Revista de Pedagogía, 63(2), 15-25. Disponible en https:// recyt.fecyt.es/index.php/BORDON/article/view/28968

Durkheim, E. (1999). Educación y Sociología. Barcelona: Altaya. 
Labaree, D. (2000). On the nature of teaching and teacher education. Difficult practices that look easy. Journal of Teacher Education, 5(3), 228-233. Available from https:// web.stanford.edu/ dlabaree/publications/Nature_of_Teaching_and_TE.pdf

López, G. (2012). Pensamiento crítico en el aula. Docencia e Investigación, (22), 41-60. Recuperado de https://www.educacion.to.uclm.es/pdf/revistaDI/3_22_2012.pdf

Maldonado, M. (2007). El trabajo colaborativo en el aula universitaria. Laurus, 13(23), 263-278. Disponible en https://revistas.upel.edu.ve/index.php/laurus/index

Maxey, C. (1956). The work of the teacher. Phi Delta Kappan, 37(6), 264-266. Available: https://www.jstor.org/stable/20341761

Noguera, J. (2002). El Concepto de Trabajo y la Teoría Social Crítica. Papers, 68, 141168. https://doi.org/10.5565/rev/papers/v68n0.1445

Polanco, A. (2005). La motivación en los estudiantes universitarios. Revista Actualidades Investigativas en Educación, 5(2), 1-13. https://doi.org/10.15517/aie.v5i2.9157

Prince, M. (2004). Does Active Learning Work? A Review of the research. Journal of Engineering Education, 93(3), 223-231. https://doi.org/10.1002/j.2168-9830.2004. tb00809.x

Robles, B. (2011). La entrevista en profundidad: una técnica útil dentro del campo antropofísico. Cuicuilco, 18(52), 39-49. Disponible en https://revistas.inah.gob.mx/ index.php/cuicuilco/article/view/3957

Valle, A. y Núñez, J. (1989). Las expectativas del profesor y su incidencia en el contexto institucional. Revista de Educación, (290), 295-319. Recuperado de https://www. educacionyfp.gob.es/dam/jcr:d3e99a20-62a1-49e1-bf7c-55f4a74d71bd/re29015-pdf. pdf

Vero, E. y Puka, E. (2018). The effectiveness of critical thinking in higher education. Online Journal Modelling the New Europe, (26), 217-233. Recuperado de http://neweurope.centre.ubbcluj.ro/wp-content/uploads/2018/06/THE-EFFECTIVENESS-OFCRITICAL-THINKING-IN-HIGHER-EDUCATION.pdf

Villa, J. (2017). La enseñanza en la universidad medieval. Centros, métodos, lecturas. Tiempo y Sociedad, (26), 59-131. Recuperado de https://tiemposociedad.files.wordpress.com/2017/01/tiempo-y-sociedad-26.pdf 
Benigno Benavides Martínez es profesor de tiempo completo de la facultad de Filosofía y Letras de la UANL. Licenciado en Sociología. Doctor en Ciencias Sociales por la Universidad Autónoma de Nuevo León (México). ORCID: https://orcid.org/0000-0002-2515-0377 